\title{
Test Method for Narrowband F/TDMA-based Wireless Sensor/Actuator Networks
}

\author{
C. Cammin 1 , D. Krush 1 , R. Heynicke ${ }^{1}$, G. Scholl', \\ ${ }^{1}$ Helmut-Schmidt-University, Holstenhofweg 85, 22043 Hamburg, Germany, \\ christoph.cammin@hsu-hh.de
}

\begin{abstract}
:
A test method for narrowband wireless sensor/actuator networks is presented, facilitating affordable and efficient performance and compliance tests prior to the deployment of the wireless communication systems. Employing frequency/time division multiple access (F/TDMA) techniques and protocol synchronization, a new and efficient test method is suggested, where the equipment under test (EUT) is evaluated under a worst-case scenario. A cost-efficient channel emulator is introduced to emulate fading in multipath environments. Also industrial interferes can be included in the setup. Furthermore, a procedure to test wireless devices with integrated, non-detachable antennas is presented.
\end{abstract}

Key words: Wireless Sensor/Actuator Network, Testing, Narrowband, TDMA, FDMA, Reverberation Chamber

\begin{abstract}
Introduction
Industrial applications pose stringent requirements on wireless systems reliability, maximum delay time, and coexistence behavior. In order to ensure a reliable wireless systems operation in the field pretests in welldefined, representative and reproducible environments have to be accomplished.
\end{abstract}

Generally, the equipment under test (EUT) has to comply with a given wireless standard so that conformity and compatibility tests have to be carried out. These tests are impeded by EUTs with a non-detachable, integrated antenna, e.g., a sealed sensor. For these EUTs the test signals have to be coupled into the EUT via radiation. Another issue is the question how the EUT will behave in the target environment, especially in a multipath environment with fading radio channels.

The purpose of a wireless standard is to guarantee system interoperability for subsystems normally produced by different manufacturers. Commonly, wireless standards also include additional rules on the equipment to provide necessary performance requirements.

Generally, the performance degradation of a wireless system can have multiple reasons, e.g. time jitter, unstable frequency, frequency offset, modulation impairments or an insufficient system dynamic range. All these imperfections influence the frame- and bit error probabilities
(FEP, BEP), which are integral indicators for the system performance.

As frequency bands for the operation of wireless devices are limited resources, many wireless communication systems have to share the same frequency bands, especially the ISM (industrial, scientific, medical) frequency band in the $2.4 \mathrm{GHz}$ range, which is one of only a few bands that is available worldwide [1]. Although coexistence mechanisms are taken into account in most wireless standards, where a competing coexistence environment often degrades the performance of a wireless system in terms of lowered data throughput and/or increased delay time [1]. Also a higher number of communication nodes might lower the performance of each single node. As a consequence the system should be tested under full communication load to be sure that there will be no performance degradation even in worst-case scenarios.

The following considerations are predominantly focused on IO-Link wireless, an emerging wireless communication standard for factory automation [2]. Without loss of generality the approach can also be applied to other communication systems as well.

Many wireless communication systems utilizes time division multiple access (TDMA) schemes for media access, whereby the radio channel is subdivided into timeslots, which are assigned to the different devices [2]. 
If low cycle times in the order of a few ms are required, a star-shaped topology with numerous (e.g. up to 60 [1] or up to 100 [2]) nodes (e.g. sensors, actuators) connected to a single base station (BS) is typically used [3]. An illustration is shown in Fig. 1.

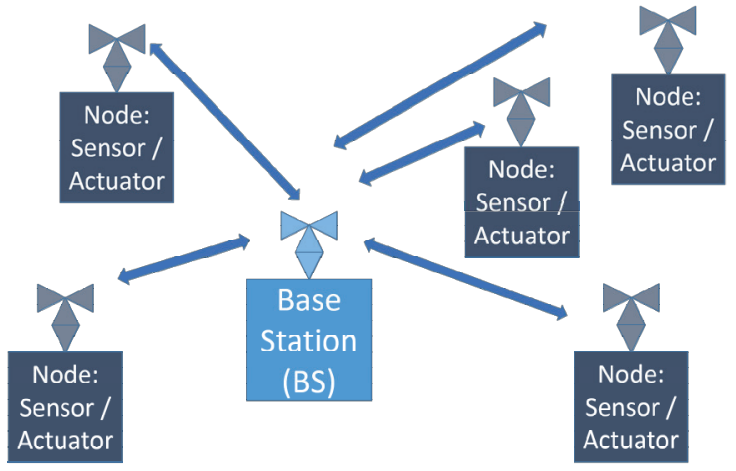

Fig. 1. Star-shaped topology: Nodes are directly connected to a base station.

\section{Indoor Radio Channel (in Industrial Environments)}

Generally, the time-variant and frequencyselective behavior of a radio channel can roughly be described by two parameters, which are coherence time and coherence bandwidth $[3,4,5]$. The first parameter describes an average temporal period, during which the radio channel remains approximately constant over time and the latter describes an average frequency spacing, within which the radio channel remains approximately constant over frequency, respectively $[4,5]$.

According to the definition in [3], in an industrial environment the coherence time typically lies between $2 \mathrm{~ms}$ and $15 \mathrm{~ms}$ and the coherence bandwidth between $6 \mathrm{MHz}$ and $60 \mathrm{MHz}$, respectively $[3,4]$.

If the coherence bandwidth is significantly larger than the transmission bandwidth of the wireless communication system the radio channel can be considered as frequency-flat and is denoted as "narrowband" channel.

The radio channel is commonly described by the complex transfer function $\underline{H}(f, t)$ of time $t$ and frequency $f$ [4]. Phase or groupdelay variations of the radio channel can be neglected in the narrowband case [4]. Without oversimplification the radio channel can thus be emulated by a specific attenuation $|\underline{H}(f, t)|$ for a particular time and frequency [4].

Communication systems like Bluetooth [4], IOLink wireless [2] and several other industrial sensor/actuator networks, e.g. [6], employ narrowband communication, where these assumptions are applicable.
Various models have been derived for the small-scale fading behavior of indoor radio channels, where Rayleigh- and Rice channel models are oftenly employed for non-line-ofsight (NLOS) or partially obstructed line-of-sight (OLOS) radio channels, respectively $[4,5]$. The Rayleigh channel model is considered as worstcase scenario, generally.

\section{F/TDMA wireless communication protocols/systems}

Fig. 2 shows a generic F/TDMA communication system. Signals from the base station towards the node are denoted as downlink signals DL1 up to DLm whereas signals from the nodes towards the BS are denoted as uplink signals UL1.1 up to ULm.n. The system in Fig.2 is a half-duplex system, as DL and UL are transmitted alternatingly.

In an F/TDMA scheme, communication is typically arranged in $\mathrm{m}$ "frequency-tracks" and $\mathrm{n}$ timeslots, as shown in Fig. 2. Additionally, a frequency hopping algorithm is oftenly used to mitigate fading effects and to improve coexistence behavior [1, 2]. Although the complete frequency band is used over time, only a few frequency-tracks are in use during a specific communication cycle.

As the focus is on narrowband systems, which typically also have a packet length well below the coherence time the transfer function of the radio channel can be simplified to $\underline{\underline{H}}\left(\mathrm{f}_{\mathrm{i}}, \mathrm{t}_{\mathrm{j}}\right) \mid$ for a particular frequency channel "i" and a particular timeslot "j". Therefore one time-variant attenuation associated to each narrowband frequency-track is sufficient to emulate the radio channel appropriately.

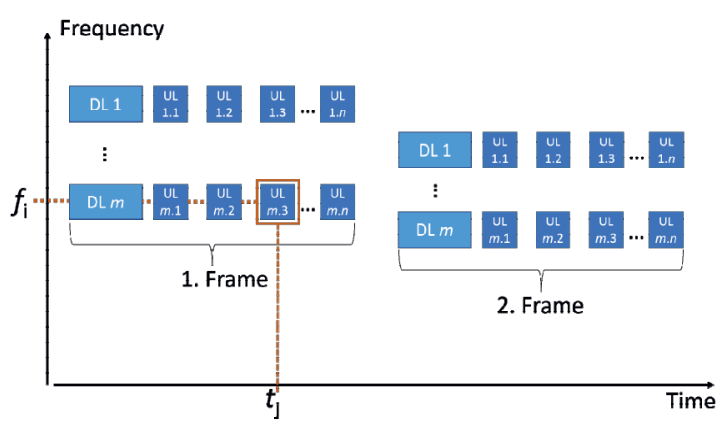

Fig. 2. Generic sketch of a (half-duplex) F/TDMA communication system. The frequencies change between the frames in this example.

\section{Test System Setup}

The concept for the test setup is based on a test equipment (TE), which comprises at least a "communication partner" for the EUT, a radio channel emulator (CE) to provide time- and frequency specific attenuation, equipment to generate the signals of other system 
subscribers (Fig. 3, Fig. 5) and components for RF signal distribution.

The CE emulates fading by applying the specific $\left|\underline{H}\left(f_{i}, t_{j}\right)\right|$. To implement the CE, synchronized, fast switching attenuators, indicated in Fig.4 and Fig.6, can be used. The synchronously switching of the attenuators to the protocol of the radio units allows to reduce the number of attenuators to one attenuator per frequency-track without oversimplification.

When testing a system under full communication load, it is not practical to deploy the maximum allowed number of discrete nodes, e.g. 60 [1] or 100 [2] nodes in a test setup. Instead, the signals of the nodes are emulated by the "test companion" (TC): Similar to an ordinary node the TC receives the DL signal from the BS and configures itself. Thus, the TC emulates other nodes by generating their UL and DL signals. Utilizing protocol synchronization, the same number of narrowband transmitters in the TC as frequency-tracks ( $m$ in Fig. 2) is sufficient to emulate the full traffic of the communication system. In contrast to alternative implementations on basis of an arbitrary / vector signal generator or on basis of highspeed digital signal processing communication testers, this approach allows to use the same narrowband transceivers as in standard nodes of the communication system. This enables a cost-efficient implementation of the TC.

With combination CE and TC and protocolsynchronization all nodes can be accurately emulated by their specific UL signals and their associated fading radio channel, i.e. $\left|\underline{H}\left(f_{i}, t_{j}\right)\right|$. Furthermore, the parallel use of narrowband transceivers has the advantage that they have independent signal chains. In conjunction with an attenuator, which has typically an operating bandwidth of several gigahertz, a faded narrowband signal can be generated easily.

Exemplary implementations of this approach including adaptions to test either a node or a BS are presented in the following sections.

\section{Node testing}

In order to provide a communication partner for the EUT, for node-testing (sensor/actuatortesting) the TE has to include a BS as well. To test the EUT as a part of the wireless communication system, all other network nodes up to the system capacity limit have to be emulated by the TC, as shown in Fig. 3. In this example the EUT is assigned to frequencytrack $\mathrm{i}$ and timeslot $\mathrm{j}$.

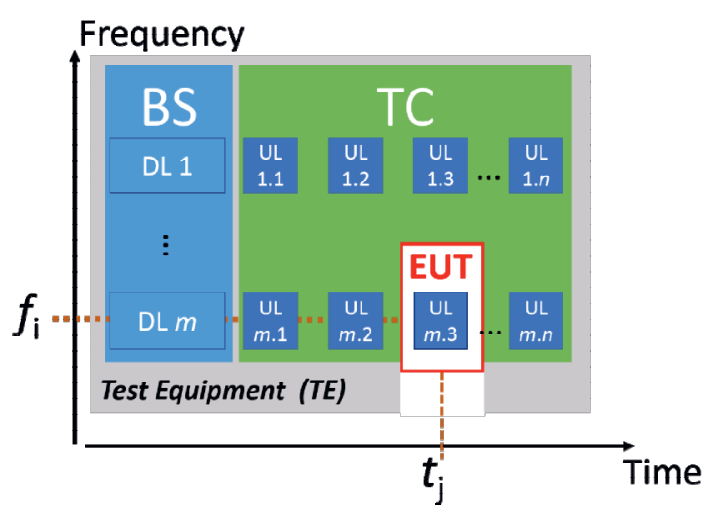

Fig. 3. Sketch of the DL and UL signals for nodetesting.

An exemplary setup for the TE is presented in Fig. 4. The TE consists of the subsystems BS, $\mathrm{TC}$ and $\mathrm{CE}$. The CE provides the associated attenuation for each UL and DL signal that is applied to the EUT. As the BS represents the master of the communication system, the overall system timing for the TDMA is provided by the BS. Therefore it is convenient to derive the synchronization for the TC and the CE from the DL signal of the BS. Optionally, further interferes (e.g. WLAN) can be added.

The whole TE is designed to accurately meet the specification of the standard and can therefore considered as "golden" equipment, according to a "golden master" in [7].

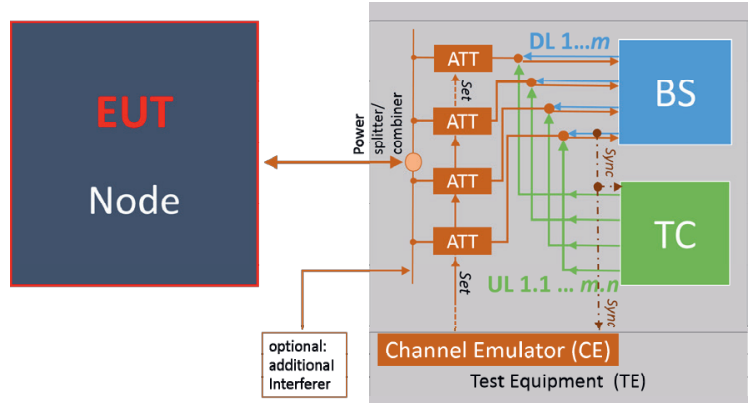

Fig. 4. General test setup for node testing.

\section{Base station testing}

Especially for BS, testing under full communication load is important. At least parts of the data handling in a BS is realized by serial processing, like, e.g. data processing or the communication over a wired network or fieldbus and thus possible bottlenecks should be identified. Here the TC is the communication partner for the EUT (BS) emulating the UL signals of all nodes, as shown in Fig. 5. 


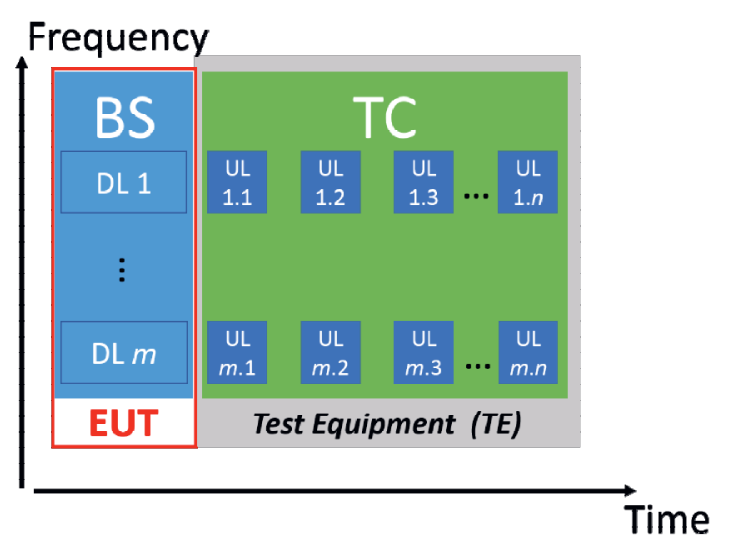

Fig. 5. Sketch of the DL and UL signals for nodetesting.

The test setup for a BS is presented in Fig. 6, where EUT (BS) acts as communication master. The TC generates the emulated UL signals from the nodes and the CE provides the specific $\left|\underline{H}\left(f_{i}, t_{j}\right)\right|$. Also the timing accuracy has to be tested by the TC and external measurement instruments. As the TC emulates node signals and provides measurement routines in this symmetric test setup the TC acts as an "inverted" BS $\left(\mathrm{BS}^{-1}\right)$ in this context. Here also the overall TE is designed to accurately meet the specification of the wireless standard and can therefore considered as "golden device" in accordance to [7].

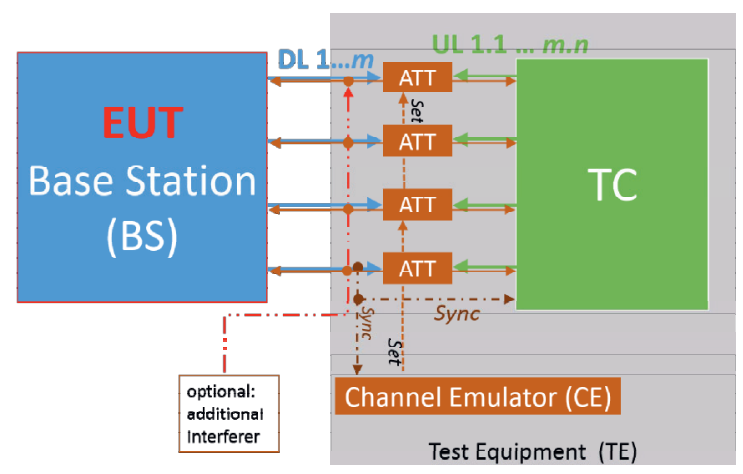

Fig. 6. Test setup for BS testing.

\section{EUT with integrated antenna}

To couple signals wirelessly into EUT with integrated antennas, several shielded test enclosures (e.g. semi/full anechoic chamber, GTEM-cell, reverberation chamber) were suggested [8]. A reverberation chamber (RC) consist of a shielded volume, which is equipped with mode-stirrers. Usually these are rotating or moving plates to stir the electromagnetic fields within the chamber step-vise or continuously [8, 9]. Besides these mechanical mode stirrers, also other types of mode-stirrers can be utilized, e.g. [10-12].
In contrast to most other test enclosures RCs provide a "stochastically isotropic" fields [8] and intrinsic radio channels with Rayleigh (or Rician) fading behavior [9, 13-17]. This means that for a full mode-stirring cycle the corresponding set of measurements results in an isotropic and homogenous environment for the EUT.

The coherence bandwidth of the RC-Rayleigh channel can be adjusted to the same values as measured in industrial environments [18-19], i.e. the intrinsic channel can be tuned to emulate a particular environment by loading the $\mathrm{RC}$ e.g. with absorbers [9, 18-22]. This allows to omit an additional CE [23].

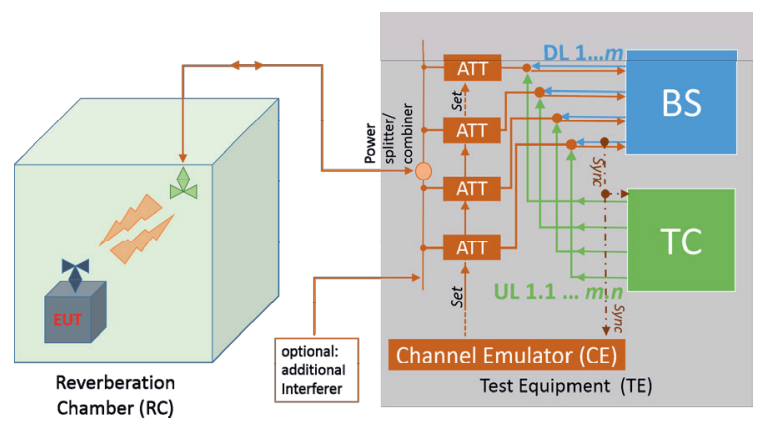

Fig. 7. Test-setup for EUT with integrated antenna.

For quality testing during the production process a simplified approach is suggested, which is similar to the "relative measurements" in [24]. First, a prototype of the series product is tested in an RC according to the approach presented above. In a second step the same prototype is mounted in a small absorber chamber, or more specifically, in a test fixture (TF) instead of a RC and the test is repeated. The measured FEP/BEP obtained in the TF can then be used as reference values. In contrast to a test in a RC, a TF does not provide a (statistically) isotropic and homogenous field distribution. But the measured reference values can be used if the EUT is always mounted in the same position and orientation within the TF.

\section{Conclusion}

A new approach to test wireless subsystems as part of an overall communication system in a fading multipath radio channel environment is presented. Exemplary embodiments for node and BS testing were presented. Utilizing protocol synchronization, the complete communication system can be efficiently emulated. Furthermore, the complexity of the TC and CE can be reduced. As a result, test costs can be significantly decreased compared to communication testers and channel emulators based on high-speed digital signal processing. 


\section{Acknowledgement}

The test equipment presented here is patented by [25].

\section{References}

[1] Cammin et al., Coexisting wireless sensor networks in cyber-physical production systems, 2016 IEEE 21 $1^{\text {st }}$ International Conference on Emerging Technologies and Factory Automation (ETFA), doi: 10.1109/ETFA.2016.7733593

[2] D. Krush, et al., Standardisierung eines schnellen drahtlosen Sensor/Aktor-Netzwerkes für die Fertigungsautomatisierung, tm - Technisches Messen. Band 83, Heft 4, 201-207 (2016), doi: 10.1515/teme-2015-0118

[3] H.J. Körber, H. Wattar, G. Scholl, Modular Wireless Real-Time Sensor/Actuator Network for Factory Automation Applications, IEEE Transactions on Industrial Informatics Vol. 3, Issue 2, 111-119 (2007); doi: 10.1109/TII.2007.898451

[4] T.S. Rappaport, Wireless Communications: Priciples and Practice, $2^{\text {nd }}$ Edition, Prentice Hall (2002), ISBN: 987-0130422323

[5] P.M. Shankar, Fading and Shadowing in Wireless Systems, Springer (2012), ISBN: 9781461403661; doi: 10.1007/978-1-4614-0367-8

[6] Krush et al., A wireless communication system for energy and environmental monitoring, Journal of Sensors and Sensor Systems (JSSS) Vol. 6, 19-26 (2017); doi: 10.5194/jsss-6-19-2017

[7] IO-Link Community, IO-Link Test Specification Version 1.1.2, 2014, [Online:] http://www.iolink.com/share/Downloads/Testspec/IOL-TestSpec_10032_V112_Jul14.pdf [last access: 01.03.2017]

[8] A.J. Schwab, W. Kürner, Elektromagnetische Verträglichkeit, 6. Auflage, Springer-Verlag, Berlin, (2011); ISBN 978-3-642-16609-9

[9] C. L. Holloway, et al., On the Use of Reverberation Chambers to Simulate a Rician Radio Environment for the Testing of Wireless Devices, IEEE Transactions on Antennas and Propagation Volume 54 Issue 11, 3167 - 3177 (2006); doi: 10.1109/TAP.2006.883987

[10] K. Rosengren et al., Characterization of Antennas for Mobile and Wireless Terminals by using Reverberation Chambers: Improved Accuracy by Platform Stirring, 2001 IEEE Antennas and Propagation Society International Symposium; doi: 10.1109/APS.2001.960105

[11] F. Moglie et al., A mechanical mode-stirred reverberation chamber inspired by chaotic cavities, 2015 IEEE Metrology for Aerospace (MetroAeroSpace); doi: 10.1109/MetroAeroSpace.2015.7180697

[12] M.S. Kildal, Investigation of mode stirring with plate on platform in a reverberation chamber, 2015 9th European Conference on Antennas and Propagation (EuCAP);
[13] J.G. Kostas, B.Boivre, Statistical model for a mode-stirred chamber, IEEE Transactions on Electromagnetic Compatibility Vol. 33, Issue 4, 1991; doi: 10.1109/15.99120

[14] P.S. Kildal et al., Cost-effective measurement setups for testing wireless communication to vehicles in reverberation chambers and anechoic chambers, 2014 IEEE Conference on Antenna Measurements \& Applications (CAMA); doi: 10.1109/CAMA.2014.7003428

[15] P. Corona, G. Ferrara, M. Migliaccio, Reverberating chamber electromagnetic field in presence of an unstirred component, IEEE Transactions on Electromagnetic Compatibility Vol. 42, Issue 2, 2000; doi: 10.1109/15.852404

[16] P.S. Kildal et al., Characterization of Reverberation Chambers for OTA Measurements of Wireless Devices: Physical Formulations of Channel Matrix and New Uncertainty Formula, IEEE Transactions on Antennas and Propagation Vol. 60, Issue 8, (2012); doi: 10.1109/TAP.2012.2201125

[17] X. Chen, P.S. Kildal, J. Carlsson, Verification of the Rician K-factor-based uncertainty model for measurements in reverberation chambers, IET Science, Measurement \& Technology Vol. 9, Issue 5, (2015); doi: 10.1049/iet-smt.2014.0344

[18] J.B. Coder et al., Examining the true effectiveness of loading a reverberation chamber: How to get your chamber consistently loaded, 2010 IEEE International Symposium on Electromagnetic Compability (EMC); doi: 10.1109/ISEMC.2010.5711332

[19] E. Genender et al., Simulating the Multipath Channel With a Reverberation Chamber: Application to Bit Error Rate Measurements, IEEE Transactions on Electromagnetic Compatibility Vol. 52, Issue 4, 766 - 777 (2010); doi: 10.1109/TEMC.2010.2044578

[20] X. Chen, P.S. Kildal, S.H. Lai, Estimation of Average Rician K-Factor and Average Mode Bandwidth in Loaded Reverberation Chamber, IEEE Antennas and Wireless Propagation Letters Vol. 10, 1437-1440 (2011); doi: 10.1109/LAWP.2011.2179910

[21] V. Rajamani, C.F. Bunting, J.C. West, Effects of loading on independent samples and uniformity of a reverberation chamber, 2013 IEEE Int. Symposium on Electromagnetic Compability (EMC); doi: 10.1109/ISEMC.2013.6670412

[22] Y. Cui, S.Y. Wang, Y. Mao, Study of the effect of chamber shape and loading position on reverberation chamber performance, 2012 AsiaPacific Symposium on Electromagnetic Compability (APEMC); doi: 10.1109/APEMC.2012.6237821

[23] C.S. Patane Lötbäck, A. Skarbratt, C. Orlenius, Extending the reverberation chamber using a channel emulator for characterisation of over-theair performance of multiple-input-multiple-output wireless devices, IET Science, Measurement \& 
Technology Vol. 9, Issue 5, 555 - 562 (2015); doi: 10.1049/iet-smt.2014.0290

[24] ETSI EN 300328 -V2.1.1, Wideband transmission systems; Data transmission equipment operating in the $2,4 \mathrm{GHz}$ ISM band and using wide band modulation techniques; (2016-11), [Online:]

http://www.etsi.org/deliver/etsi_en/300300 30039 9/300328/02.01.01_60/en_300328v020101p.pdf [last access: 05.03.2017]

[25] Patent EP 3073655 A1, Kanalemulator und Prüfsystem für Kommunikationsteilnehmer 